\title{
The Size of Generating Sets of Powers
}

\author{
Dmitriy Zhuk
}

\begin{abstract}
In the paper we prove for every finite algebra $\mathbb{A}$ that either it has the polynomially generated powers (PGP) property, or it has the exponentially generated powers (EGP) property. For idempotent algebras we give a simple criteria for the algebra to satisfy EGP property.
\end{abstract}

\section{Main Results}

We say that an algebra $\mathbb{A}$ has the polynomially generated powers (PGP) property if its $n$-th power $\mathbb{A}^{n}$ has a polynomial-size generating set. That is, there exists a polynomial $p$ such that for every $n$ the $n$-th power $\mathbb{A}^{n}$ can be generated by at most $p(n)$ tuples. We say that an algebra $\mathbb{A}$ has the exponentially generated powers (EGP) property if there exists $b>1$ and $C>0$ such that for every $n$ the $n$-th power $\mathbb{A}^{n}$ cannot be generated by less than $C b^{n}$ tuples.

Theorem 1. Suppose $\mathbb{A}$ is a finite algebra. Then either $\mathbb{A}$ has PGP property, or $\mathbb{A}$ has EGP property.

Suppose $\alpha, \beta \subsetneq A, \alpha \cup \beta=A$. We say that an operation $f: A^{n} \rightarrow A$ is $\alpha \beta$-projective if there exists $j \in\{1,2, \ldots, n\}$ such that for every $\left(a_{1}, \ldots, a_{n}\right) \in A^{n}$ and $S \in\{\alpha, \beta\}$ we have $f\left(a_{1}, \ldots, a_{j-1}, S, a_{j+1}, \ldots, a_{n}\right) \subseteq S$.

Theorem 2. Suppose $\mathbb{A}$ is a finite idempotent algebra. Then $\mathbb{A}$ has EGP property if and only if there exist $\alpha$ and $\beta$ such that every operation of the algebra is $\alpha \beta$-projective.

For an algebra $\mathbb{A}$ and a subset $X \subset A^{n}$, by $\langle X\rangle_{\mathbb{A}}$ we denote the subalgebra generated by $X$. For an integer $m$ put $D_{A, m}=\left\{\left(a_{1}, \ldots, a_{2 m}\right) \mid\left(a_{1}=a_{2}\right) \vee\left(a_{3}=a_{4}\right) \vee \cdots \vee\left(a_{2 m-1}=a_{2 m}\right)\right\}$.

Theorem 3. Suppose $\mathbb{A}$ is a finite algebra. Then $\mathbb{A}$ has EGP property if and only if $\left\langle D_{A, m}\right\rangle_{\mathbb{A}} \neq$ $A^{2 m}$ for every $m \geqslant|A|$.

\section{Proof of Theorem 1 and Theorem 3}

For a tuple $\left(a_{1}, \ldots, a_{n}\right)$ we say that $i \in\{1,2, \ldots, n-1\}$ is a switch if $a_{i} \neq a_{i+1}$. An algebra is called $r$-switchable, if $\mathbb{A}^{n}$ is generated by all tuples of length $n$ with at most $r$ switches. $\mathbb{A}$ is called switchable if $\mathbb{A}$ is $r$-switchable for some $r$. We assume that $A=\{0,1, \ldots, k-1\}$. A relation $\rho \subset A^{m}$ is called nice if $\rho$ is not full and for all $c_{1}, \ldots, c_{m} \in A$

$$
\left(\exists i: c_{i}=c_{i+1}\right) \Rightarrow\left(c_{1}, \ldots, c_{m}\right) \in \rho .
$$

By $\operatorname{Inv}(\mathbb{A})$ we denote all invariant relations of an algebra $\mathbb{A}$.

It is easy to check the following lemma 
Lemma 4. Suppose a finite algebra $\mathbb{A}$ is switchable, then it has PGP property.

Lemma 5. Suppose a finite algebra $\mathbb{A}$ is not switchable. Then for every $r$ there exists a nice relation in $\operatorname{Inv}(\mathbb{A})$ of arity $m \geqslant r$.

Proof. Since $\mathbb{A}$ is not switchable, for every $r$ there exists $n$ such that $\mathbb{A}^{n}$ is not generated by all tuples with at most $r$ switches. Let $H \subseteq A^{n}$ be the set of all tuples with at most $r$ switches. Put $\sigma=\langle H\rangle_{\mathbb{A}}$. Let $\alpha$ be a tuple in $A^{n} \backslash \sigma$ with the minimal number of switches. Suppose

$$
\alpha=(\underbrace{a_{1}, \ldots, a_{1}}_{n_{1}}, \underbrace{a_{2}, \ldots, a_{2}}_{n_{2}}, \ldots, \underbrace{a_{m}, \ldots, a_{m}}_{n_{m}}),
$$

where $a_{1} \neq a_{2}, a_{2} \neq a_{3}, \ldots, a_{m-1} \neq a_{m}$. By the definition, $m-1>r$. Put

$$
\rho\left(x_{1}, \ldots, x_{m}\right)=\sigma(\underbrace{x_{1}, \ldots, x_{1}}_{n_{1}}, \underbrace{x_{2}, \ldots, x_{2}}_{n_{2}}, \ldots, \underbrace{x_{m}, \ldots, x_{m}}_{n_{m}}) .
$$

Since $\sigma$ contains all tuple with at most $m-2$ switches, $\rho$ is nice. This completes the proof.

Lemma 6. Suppose $\mathbb{A}$ is a finite algebra, $\rho \in \operatorname{Inv}(\mathbb{A})$ is a nice relation of arity $m$ such that $m>2 k^{2} \cdot n^{2}$. Then there exists a relation $\sigma \in \operatorname{Inv}(\mathbb{A})$ of arity $2 n+k$ such that $\sigma$ is not full and for all $c_{1}, \ldots, c_{n}, d_{1}, \ldots, d_{n}, e_{1}, \ldots, e_{k} \in A$ we have

$$
\left(\exists i, j \in\{1,2, \ldots, n\}: c_{i}=d_{j}\right) \Rightarrow\left(c_{1}, \ldots, c_{n}, d_{1}, \ldots, d_{n}, e_{1}, \ldots, e_{k}\right) \in \sigma .
$$

Proof. Since $\rho$ is not full, there exists a tuple $\left(a_{1}, \ldots, a_{m}\right) \in A^{m} \backslash \rho$. We consider the sequence of pairs $\left(a_{1}, a_{2}\right),\left(a_{3}, a_{4}\right),\left(a_{5}, a_{6}\right), \ldots$, where the last pair is $\left(a_{m-1}, a_{m}\right)$ if $m$ is even and $\left(a_{m-2}, a_{m-1}\right)$ if $m$ is odd. We choose the most popular pair in the sequence. Suppose this pair is $(a, b)$ and it appears $l \geqslant n^{2}$ times. Then we identify variables in the relation $\rho$ as follows

$$
\delta\left(x_{1}, y_{1}, x_{2}, y_{2}, \ldots, x_{l}, y_{l}, z_{0}, \ldots, z_{k-1}\right)=\rho\left(t_{1}, \ldots, t_{m}\right),
$$

where $t_{i}=x_{j}$ and $t_{i+1}=y_{j}$ if $\left(a_{i}, a_{i+1}\right)$ is the $j$-th pair in the sequence that is equal to $(a, b)$; and $t_{i}=z_{a_{i}}$ otherwise. We can easily see that $(a, b, a, b, a, b, \ldots, a, b, 0,1, \ldots, k-1) \notin \delta$. It remains to define a relation $\sigma$. This time we identify variables as follows

$$
\sigma\left(x_{1}, \ldots, x_{n}, y_{1}, \ldots, y_{n}, z_{0}, \ldots, z_{k-1}\right)=\delta\left(r_{1}, s_{1}, r_{2}, s_{2}, \ldots, r_{l}, s_{l}, z_{0}, \ldots, z_{k-1}\right),
$$

where $r_{i} \in\left\{x_{1}, \ldots, x_{n}\right\}$ for every $i, s_{i} \in\left\{y_{1}, \ldots, y_{n}\right\}$ for every $i$, and the pair $\left(x_{i}, y_{j}\right)$ appears at least once among the pairs $\left(r_{1}, s_{1}\right),\left(r_{2}, s_{2}\right), \ldots,\left(r_{l}, s_{l}\right)$ for every $i$ and $j$. Since $l \geqslant n^{2}$, we always can do this. We know that $(\underbrace{a, a, \ldots, a}_{n}, \underbrace{b, b, \ldots, b}_{n}, 0,1,2, \ldots, k-1) \notin \sigma$, hence $\sigma$ satisfies the condition of the lemma.

Theorem 7. Suppose $\mathbb{A}$ is a finite algebra. Then either $\mathbb{A}$ is switchable, or $\mathbb{A}$ has EGP property.

Proof. Suppose $\mathbb{A}$ is not switchable. Then by Lemma 5 for every $r$ there exists a nice relation of arity $m^{\prime} \geqslant r$ in $\operatorname{Inv}(\mathbb{A})$. Then by Lemma 6 for every $n$ there exists a relation $\sigma \in \operatorname{Inv}(\mathbb{A})$ of arity $2 n+k$ such that $\sigma$ is not full and for all $c_{1}, \ldots, c_{n}, d_{1}, \ldots, d_{n}, e_{1}, \ldots, e_{k} \in A$

$$
\left(\exists i, j \in\{1,2, \ldots, n\}: c_{i}=d_{j}\right) \Rightarrow\left(c_{1}, \ldots, c_{n}, d_{1}, \ldots, d_{n}, e_{1}, \ldots, e_{k}\right) \in \sigma .
$$

Let us consider all relations that can be obtained from $\sigma$ by a permutation of the first $2 n$ variables. The family of all such relations we denote by $\Sigma$. Obviously, it contains exactly $2 n$ ! 
relations. Assume that $\mathbb{A}^{2 n+k}$ is generated by tuples $\alpha_{1}, \ldots, \alpha_{s}$. Since all relations in $\Sigma$ are not full, for every relation $\sigma^{\prime} \in \Sigma$ there exists $i \in\{1,2, \ldots, s\}$ such that $\alpha_{i} \notin \sigma^{\prime}$.

Let us count how many relations from $\Sigma$ can omit a tuple $\left(a_{1}, \ldots, a_{2 n+k}\right)$. Suppose

$$
\sigma^{\prime}\left(t_{1}, \ldots, t_{2 n}, z_{0}, \ldots, z_{k-1}\right)=\sigma\left(x_{1}, \ldots, x_{n}, y_{1}, \ldots, y_{n}, z_{0}, \ldots, z_{k-1}\right)
$$

where $\left\{t_{1}, \ldots, t_{2 n}\right\}=\left\{x_{1}, \ldots, x_{n}, y_{1}, \ldots, y_{n}\right\}$, and $\sigma^{\prime}$ omits the tuple $\left(a_{1}, \ldots, a_{2 n+k}\right)$. Obviously, if $a_{i}=a_{j}$ then either $\left\{t_{i}, t_{j}\right\} \subseteq\left\{x_{1}, \ldots, x_{n}\right\}$, or $\left\{t_{i}, t_{j}\right\} \subseteq\left\{y_{1}, \ldots, y_{n}\right\}$. Therefore, to define a permutation of variables it is sufficient to define a mapping $A \rightarrow\{x, y\}$, and the order of $x$ 's and $y$ 's in $t_{1}, \ldots, t_{2 n}$. We conclude that a tuple can be omitted by at most $n ! \cdot n ! \cdot 2^{k}$ relations. Hence $s \geqslant(2 n !) /\left((n !)^{2} \cdot 2^{k}\right)>2^{n-k}$. This means that for every $m$ we need at least $2^{[(m-k) / 2]-k} \geqslant 2^{m / 2-2 k}$ tuples to generate $\mathbb{A}^{m}$. It remains to put $b=\sqrt{2}$ and $C=2^{-2 k}$ in the definition of EGP property

Theorem 1 follows from Lemma 4 and Theorem 7.

Lemma 8. Suppose $\mathbb{A}$ is a finite algebra, $m \geqslant k,\left\langle D_{A, m}\right\rangle_{\mathbb{A}}=A^{2 m}$, then $\left\langle D_{A, n}\right\rangle_{\mathbb{A}}=A^{2 n}$ for every $n>m$.

Proof. Suppose $\alpha=\left(a_{1}, \ldots, a_{2 n}\right) \in A^{2 n}$. Put $\alpha^{\prime}=\left(a_{1}, \ldots, a_{2 m}\right)$. Let us show that $\alpha \in$ $\left\langle D_{A, n}\right\rangle_{\mathbb{A}}$. We know that there exist tuples $\gamma_{1}^{\prime}, \ldots, \gamma_{s}^{\prime} \in D_{A, m}$ and a term operation $f$ such that $f\left(\gamma_{1}^{\prime}, \ldots, \gamma_{s}^{\prime}\right)=\alpha^{\prime}$.

Since $n>m \geqslant k$, WLOG we can assume that $\left\{a_{1}, \ldots, a_{2 m}\right\}=\left\{a_{1}, \ldots, a_{2 n}\right\}$. Obviously, any extension of $\gamma_{i}$ with $2 n-2 m$ elements is a tuple from $D_{A, n}$. Then, we can find extensions $\gamma_{1}, \ldots, \gamma_{s}$ such that $f\left(\gamma_{1}, \ldots, \gamma_{s}\right)=\alpha$. Hence, $\alpha \in\left\langle D_{A, n}\right\rangle_{\mathbb{A}}$.

Now, let us prove Theorem 3.

Proof. Suppose $\mathbb{A}$ is not switchable. Then by Lemma 5 for every $r \geqslant 2 k$ there exists a nice relation $\sigma$ of arity $m \geqslant r$ in $\operatorname{Inv}(\mathbb{A})$.

We want $m$ to be even. If it is odd we do the following. Suppose $\left(a_{1}, \ldots, a_{m}\right) \notin \sigma$, then some element of $A$ occurs at least twice in the sequence $a_{1}, a_{3}, a_{5}, \ldots, a_{m}$. Assume that $a_{2 j+1}=a_{2 i+1}$, where $j<i$. Then we identify the $2 j+1$-th variable and the $2 i+1$-th variable of $\sigma$ to get a relation $\sigma^{\prime}$ of arity $m^{\prime}=m-1$.

$$
\sigma^{\prime}\left(x_{1}, \ldots, x_{2 i}, x_{2 i+2}, \ldots, x_{m}\right)=\sigma\left(x_{1}, \ldots, x_{2 i}, x_{2 j+1}, x_{2 i+2}, \ldots, x_{m}\right) .
$$

If $m$ is even, we put $\sigma^{\prime}=\sigma$ and $m^{\prime}=m$. We can check that $D_{A, m^{\prime} / 2} \subseteq \sigma^{\prime}$, therefore $\left\langle D_{A, m^{\prime} / 2}\right\rangle_{\mathbb{A}} \neq A^{m^{\prime}}$. Using Lemma 8, we prove that $\left\langle D_{A, n}\right\rangle_{\mathbb{A}} \neq A^{2 n}$ for every $n \geqslant k$.

Suppose $\left\langle D_{A, m}\right\rangle_{\mathbb{A}} \neq A^{2 m}$ for every $m \geqslant k$. Then for every $m \geqslant k$ we can define a relation $\sigma_{m}=\left\langle D_{A, m}\right\rangle_{\mathbb{A}}$, which has all properties of a nice relation we use in Lemma 6. Thus, arguing as in Theorem 7 we can prove that $\mathbb{A}$ has EGP property.

\section{Criteria For the Idempotent Case}

Lemma 9. Suppose $\mathbb{A}$ is a finite algebra, $\varnothing \neq B \subsetneq A,\left\langle A^{n} \backslash(A \backslash B)^{n}\right\rangle_{\mathbb{A}} \neq A^{n}$ for every $n$, then there exists $C \subsetneq A$ such that $B \subseteq C$ and $A^{n} \backslash(A \backslash C)^{n} \in \operatorname{Inv}(\mathbb{A})$ for every $n$.

Proof. Let $C \subsetneq A$ be a maximal set containing $B$ such that $\left\langle A^{n} \backslash(A \backslash C)^{n}\right\rangle_{\mathbb{A}} \neq A^{n}$ for every $n$. Put $\sigma_{n}=\left\langle A^{n} \backslash(A \backslash C)^{n}\right\rangle_{\mathbb{A}}$, let us show that $\sigma_{n}=A^{n} \backslash(A \backslash C)^{n}$ for every $n$. Assume the converse. Then there exists $\left(a_{1}, \ldots, a_{n}\right) \in \sigma_{n} \cap(A \backslash C)^{n}$. Since the algebra is idempotent, $\left(a_{1}, \ldots, a_{n}\right) \times A^{s} \subseteq \sigma_{n+s}$ for every $s \geqslant 0$. Let $m \in\{0,1, \ldots, n-1\}$ be the 
maximal number such that $\left(a_{1}, \ldots, a_{m}\right) \times A^{s} \nsubseteq \sigma_{m+s}$ for every $s \geqslant 0$. Then for some $s^{\prime}$ we have $\left(a_{1}, \ldots, a_{m+1}\right) \times A^{s^{\prime}} \subseteq \sigma_{m+s^{\prime}+1}$. Since the algebra is idempotent, $\left(a_{1}, \ldots, a_{m+1}\right) \times A^{s} \subseteq \sigma_{m+s+1}$ for every $s \geqslant s^{\prime}$. Put

$$
\delta_{s+1}\left(x_{1}, \ldots, x_{s+1}\right)=\sigma_{m+s+1}\left(a_{1}, \ldots, a_{m}, x_{1}, \ldots, x_{s+1}\right) .
$$

By the definition of $m$ we know that $\delta_{s+1}$ is not a full relation. Since $\sigma_{m+s+1}$ is symmetric, $A^{s+1} \backslash\left(A \backslash\left\{a_{m+1}\right\}\right)^{s+1} \subseteq \delta_{s+1}$. Put $C^{\prime}=C \cup\left\{a_{m+1}\right\}$. By the definition we have $\delta_{s} \in \operatorname{Inv}(\mathbb{A})$ and $A^{s+1} \backslash\left(A \backslash C^{\prime}\right)^{s+1} \subseteq \delta_{s+1}$. Therefore, $\left\langle A^{n} \backslash\left(A \backslash C^{\prime}\right)^{n}\right\rangle_{\mathbb{A}} \subseteq \delta_{n} \neq A^{n}$ for every $n \geqslant s^{\prime}+1$. It is easy to check that the above condition holds for $n<s^{\prime}+1$. This contradicts our assumption about the maximality of $C$.

Lemma 10. Suppose $\mathbb{A}$ is a finite idempotent algebra satisfying EGP property. Then there exists a binary reflexive relation $\rho$ such that $\rho$ is not full and the relation $\sigma_{n}$ defined by

$$
\sigma_{n}\left(x_{1}, \ldots, x_{2 n}\right)=\rho\left(x_{1}, x_{2}\right) \vee \rho\left(x_{3}, x_{4}\right) \vee \cdots \vee \rho\left(x_{2 n-1}, x_{2 n}\right)
$$

is an invariant for $\mathbb{A}$ for every $n$.

Proof. Let us consider an algebra $\mathbb{D}=\mathbb{A} \times \mathbb{A}$. Put $B=\{(a, a) \mid a \in A\}$. By Theorem 3 , $\left\langle D^{n} \backslash(D \backslash B)^{n}\right\rangle_{\mathbb{A}} \neq D^{n}$ for every $n$. By Lemma 9, there exists $C \subseteq D=A \times A$ such that $D^{n} \backslash(D \backslash C)^{n} \in \operatorname{Inv}(\mathbb{D})$ for every $n$. It remains to put $\rho=C$.

Lemma 11. Suppose $\mathbb{A}$ is a finite idempotent algebra satisfying EGP property. Then there exist $\alpha, \beta \subsetneq A$ such that $\alpha \cup \beta=A, \rho=(\alpha \times \alpha) \cup(\beta \times \beta)$, and the relation $\sigma_{n}$ defined by

$$
\sigma_{n}\left(x_{1}, \ldots, x_{2 n}\right)=\rho\left(x_{1}, x_{2}\right) \vee \rho\left(x_{3}, x_{4}\right) \vee \cdots \vee \rho\left(x_{2 n-1}, x_{2 n}\right)
$$

is an invariant for $\mathbb{A}$ for every $n$.

Proof. By Lemma 10 there exists a reflexive relation $\rho_{0}$ such that the relation $\sigma_{n, 0}\left(x_{1}, \ldots, x_{2 n}\right)=$ $\rho_{0}\left(x_{1}, x_{2}\right) \vee \rho_{0}\left(x_{3}, x_{4}\right) \vee \cdots \vee \rho_{0}\left(x_{2 n-1}, x_{2 n}\right)$ is an invariant for every $n$.

First, we want $\rho_{0}$ to be a symmetric relation. Put $\rho_{0}^{\prime}(x, y)=\rho_{0}(y, x)$. We consider $2^{n}$ relations that can be obtained from $\sigma_{n, 0}$ by replacing some $\rho_{0}$ by $\rho_{0}^{\prime}$ in the definition of $\sigma_{n, 0}$. The intersection of these relations we denote by $\sigma_{n, 1}$. We can easily check that $\sigma_{n, 1}$ is an invariant for $\mathbb{A}$ and $\sigma_{n, 1}\left(x_{1}, \ldots, x_{2 n}\right)=\rho_{1}\left(x_{1}, x_{2}\right) \vee \rho_{1}\left(x_{3}, x_{4}\right) \vee \cdots \vee \rho_{1}\left(x_{2 n-1}, x_{2 n}\right)$, where $\rho_{1}=\rho_{0} \cap \rho_{0}^{\prime}$.

Assume that $\rho_{1}$ is a maximal symmetric reflexive relation such that the above relation $\sigma_{n, 1}$ is an invariant for every $n$. Let $C \subseteq A$ be the set of all elements $c$ such that $\{c\} \times A \subseteq \rho_{1}$. Put $B=A \backslash C$. Let us consider the relation $\rho_{1}^{\prime}=\rho_{1} \cap(B \times B)$. Assume that $\rho_{1}^{\prime}$ is not transitive, then for some $a, b, c \in A$ we have $(a, b),(b, c) \in \rho_{1}^{\prime}$ and $(a, c) \notin \rho_{1}^{\prime}$.

Put $\rho_{2}=\left(\rho_{1}\left(x_{1}, b\right) \vee \rho_{1}\left(x_{1}, x_{2}\right)\right) \wedge\left(\rho_{1}\left(x_{2}, b\right) \vee \rho_{1}\left(x_{1}, x_{2}\right)\right)$ and

$$
\sigma_{n, 2}\left(x_{1}, \ldots, x_{2 n}\right)=\rho_{2}\left(x_{1}, x_{2}\right) \vee \rho_{2}\left(x_{3}, x_{4}\right) \vee \cdots \vee \rho_{2}\left(x_{2 n-1}, x_{2 n}\right) .
$$

It can be easily checked that $\sigma_{n, 2} \in \operatorname{Inv}(\mathbb{A})$. Since $b \notin C$, there exists $d \in B$ such that $(b, d) \notin \rho_{1}$. Obviously, $(b, d) \notin \rho_{2}$, hence $\rho_{2}$ is not full. Also, $\rho_{2}$ is a symmetric reflexive relation such that $\rho_{1} \subsetneq \rho_{2}$. This contradicts our assumption about the maximality of $\rho_{1}$. Therefore, $\rho_{1}^{\prime}$ is transitive, and therefore, it is an equivalence relation on $B$. Assume that there are at least 3 different equivalence classes. We choose some element $b \in B$ and then define $\rho_{2}$ as above. We can easily see that $\rho_{2}$ is not full, which contradicts the maximality of $\rho_{1}$. Hence there are exactly 2 equivalence classes. This completes the proof. 
Lemma 12. Suppose $\alpha, \beta \subsetneq A, \alpha \cup \beta=A, \rho=(\alpha \times \alpha) \cup(\beta \times \beta)$, the relation $\sigma_{n}$ is defined by

$$
\sigma_{n}\left(x_{1}, \ldots, x_{2 n}\right)=\rho\left(x_{1}, x_{2}\right) \vee \rho\left(x_{3}, x_{4}\right) \vee \cdots \vee \rho\left(x_{2 n-1}, x_{2 n}\right) .
$$

Then an idempotent operation $f$ is $\alpha \beta$-projective if and only if it preserves $\sigma_{n}$ for every $n$.

Proof. Suppose $f$ is $\alpha \beta$-projective. Let $\gamma_{1}, \ldots, \gamma_{s} \in \sigma_{n}$. We need to prove that $f\left(\gamma_{1}, \ldots, \gamma_{s}\right)=$ $\delta \in \sigma_{n}$. Choose $j$ in the definition of $\alpha \beta$-projectiveness. Since $\gamma_{j} \in \sigma_{n}$ we have $\left(\gamma_{j}(2 r-\right.$ $\left.1), \gamma_{j}(2 r)\right) \in \rho$ for some $r$. Hence $\gamma_{j}(2 r-1), \gamma_{j}(2 r) \in \alpha$ or $\gamma_{j}(2 r-1), \gamma_{j}(2 r) \in \beta$. By the definition of $\alpha \beta$-projectiveness, we obtain $\delta(2 r-1), \delta(2 r) \in \alpha$ or $\delta(2 r-1), \delta(2 r) \in \beta$. Hence $\delta \in \sigma_{n}$.

Suppose $f$ of arity $s$ preserves $\sigma_{n}$ for every $n$. Assume that $f$ is not $\alpha \beta$-projective. Hence for every $j \in\{1,2, \ldots, s\}$ there exists a tuple $\left(a_{1}^{(j)}, \ldots, a_{s}^{(j)}\right)$ and $S_{j} \in\{\alpha, \beta\}$ such that $a_{j}^{(j)} \in S_{j}$ and $f\left(a_{1}^{(j)}, \ldots, a_{n}^{(j)}\right) \notin S_{j}$. Choose $S_{j}^{\prime}$ such that $\left\{S_{j}, S_{j}^{\prime}\right\}=\{\alpha, \beta\}$, and $c_{j} \in S_{j} \backslash S_{j}^{\prime}$. The following statement gives a contradiction and completes the proof

$$
f\left(\begin{array}{cccc}
a_{1}^{(1)} & a_{2}^{(1)} & \ldots & a_{s}^{(1)} \\
c_{1} & c_{1} & \ldots & c_{1} \\
a_{1}^{(2)} & a_{2}^{(2)} & \ldots & a_{s}^{(2)} \\
c_{2} & c_{2} & \ldots & c_{2} \\
\vdots & \vdots & \ddots & \vdots \\
a_{1}^{(s)} & a_{2}^{(s)} & \ldots & a_{s}^{(s)} \\
c_{s} & c_{s} & \ldots & c_{s}
\end{array}\right) \notin \sigma_{s} .
$$

Theorem 2 follows from Theorem 3, Lemma 11, and Lemma 12. 